\title{
Correction to: Bridging the gap between will and action on climate change adaptation in large cities in Brazil
}

\author{
Gabriela Marques Di Giulio ${ }^{1} \cdot$ Roger Rodrigues Torres ${ }^{2} \cdot$ David M. Lapola $^{3} \cdot$ Ana Maria Bedran-Martins $^{4}$. \\ Maria da Penha Vasconcellos ${ }^{4}$. Diego Rafael Braga ${ }^{3}$. Marcos Paulo Fuck ${ }^{5}$ - Yohanna Juk ${ }^{5}$. Veruska Nogueira ${ }^{2}$. \\ Ana Carolina Penna ${ }^{2} \cdot$ Tiago Jacaúna $^{6} \cdot$ Marcelo Fetz $^{7} \cdot$ Zoraide Pessoa $^{8} \cdot$ Rylanneive Pontes $^{8} \cdot$ Marize Schons $^{9}$. \\ Adriano Premebida ${ }^{9}$
}

Published online: 25 November 2019

(C) Springer-Verlag GmbH Germany, part of Springer Nature 2019

\section{Correction to: Regional Environmental Change https://doi.org/10.1007/s10113-019-01570-z}

Unfortunately, the paper by Di Giulio et al. 2019 (https://doi. org/10.1007/s10113-019-01570-z) contained several errors in the figure captions. Here, the figures are reprinted with corrected captions:

The online version of the original article can be found at https://doi.org/ 10.1007/s10113-019-01570-Z

Gabriela Marques Di Giulio

ggiulio@usp.br

Roger Rodrigues Torres

roger.torres@unifei.edu.br

David M. Lapola

dmlapola@unicamp.br

Ana Maria Bedran-Martins

bedran.ana@gmail.com

Maria da Penha Vasconcellos

mpvascon@usp.br

Diego Rafael Braga

diego1987@gmail.com

Marcos Paulo Fuck

marcospaulofk@gmail.com

Yohanna Juk

yohannajuk91@gmail.com

\author{
Veruska Nogueira \\ veruska@cbhsapucai.org.br \\ Ana Carolina Penna \\ acarol.penna@gmail.com \\ Tiago Jacaúna \\ tiagojacauna@ufam.edu.br \\ Marcelo Fetz \\ marcelofetz@gmail.com \\ Zoraide Pessoa \\ zoraidesp@gmail.com \\ Rylanneive Pontes \\ pontesrylanneive@gmail.com \\ Marize Schons \\ marizeschons426@gmail.com \\ Adriano Premebida \\ premebida@hotmail.com
}

Extended author information available on the last page of the article 


\section{URBAN CLIMATE ADAPTATION}

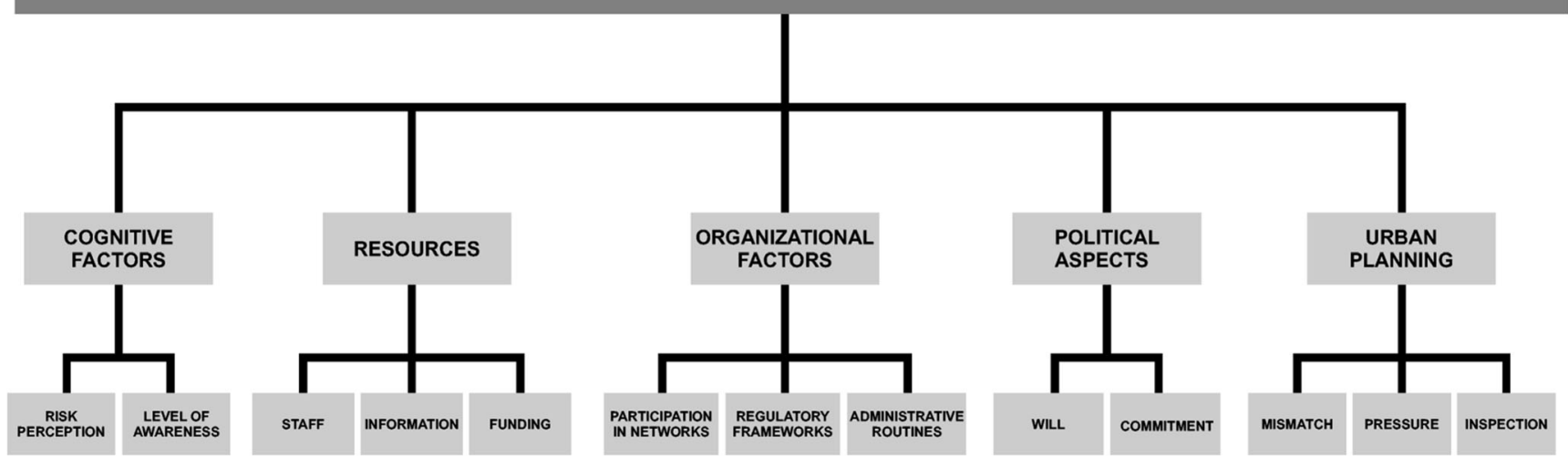

Fig. 1 The framework includes five dimensions and 13 elements that affect the ability of local governments to advance in adaptation

\begin{tabular}{|c|c|c|c|c|c|c|c|}
\hline \multicolumn{2}{|c|}{ Framework } & \multicolumn{6}{|c|}{$\begin{array}{l}\text { Assessing constraints for adaptation across } \\
\text { the municipal level }\end{array}$} \\
\hline $\begin{array}{l}\text { Dimensions that affect the } \\
\text { ability of local government to } \\
\text { advance in adaptation }\end{array}$ & Main elements & SPO & VIT & POA & CUR & NAT & MAN \\
\hline \multirow{2}{*}{$\begin{array}{l}\text { Dimension } 1 \text { - Cognitive factors } \\
\text { (to motivate climate adaptation) }\end{array}$} & risk perception & + & + & + & + & + & + \\
\hline & level of awareness & + & + & + & + & + & + \\
\hline \multirow{3}{*}{$\begin{array}{l}\text { Dimension } 2 \text { - Resources } \\
\text { (to advance the adaptation } \\
\text { agenda) }\end{array}$} & staff & +++ & +++ & ++ & +++ & ++ & ++ \\
\hline & information & +++ & ++ & ++ & ++ & +++ & ++ \\
\hline & funding & ++ & ++ & +++ & ++ & ++ & ++ \\
\hline \multirow{3}{*}{$\begin{array}{l}\text { Dimension } 3 \text { - Organizational } \\
\text { factors } \\
\text { (to deploy resources and integrate } \\
\text { adaptation as a central theme) }\end{array}$} & participation in climate networks & + & + & + & + & + & + \\
\hline & climate regulatory frameworks & + & $(-)$ & $(-)$ & $(-)$ & $(-)$ & + \\
\hline & administrative routines/practices & +++ & +++ & +++ & +++ & +++ & +++ \\
\hline \multirow{2}{*}{$\begin{array}{l}\text { Dimension } 4 \text { - Political aspects } \\
\text { (to implement climate initiatives) }\end{array}$} & political will & +++ & +++ & +++ & +++ & +++ & +++ \\
\hline & level of commitment & +++ & +++ & +++ & +++ & +++ & +++ \\
\hline \multirow{4}{*}{$\begin{array}{l}\text { Dimension } 5 \text { - Local dynamics of } \\
\text { urban planning } \\
\text { (to consolidate adaptation } \\
\text { interventions) }\end{array}$} & $\begin{array}{l}\text { mismatch between the scale of urban } \\
\text { issues and the extent of local }\end{array}$ & +++ & +++ & +++ & +++ & +++ & +++ \\
\hline & government authority & & & & & & \\
\hline & pressures from private sector & +++ & +++ & +++ & +++ & +++ & +++ \\
\hline & inspection & +++ & +++ & +++ & +++ & +++ & +++ \\
\hline
\end{tabular}

+ low impact; ++ moderate impact; +++ high impact; (-) no existence

Fig. 2 Summary of application of the framework and qualitative assessment in the six cities studied 


\section{Affiliations}

Gabriela Marques Di Giulio ${ }^{1} \cdot$ Roger Rodrigues Torres ${ }^{2} \cdot$ David M. Lapola $^{3} \cdot$ Ana Maria Bedran-Martins ${ }^{4}$. Maria da Penha Vasconcellos ${ }^{4}$. Diego Rafael Braga ${ }^{3}$. Marcos Paulo Fuck ${ }^{5}$. Yohanna Juk ${ }^{5}$. Veruska Nogueira ${ }^{2}$. Ana Carolina Penna ${ }^{2} \cdot$ Tiago Jacaúna $^{6} \cdot$ Marcelo Fetz $^{7} \cdot$ Zoraide Pessoa $^{8} \cdot$ Rylanneive Pontes $^{8} \cdot$ Marize Schons $^{9}$. Adriano Premebida ${ }^{9}$

1 School of Public Health, University of São Paulo (USP), Av. Dr. Arnaldo, 715, São Paulo, SP 01246-904, Brazil

2 Federal University of Itajubá, Itajubá, Brazil

3 University of Campinas, Campinas, Brazil

4 University of São Paulo, São Paulo, Brazil

5 Federal University of Paraná, Curitiba, Brazil
$6 \quad$ Federal University of Amazonas, Manaus, Brazil

7 Federal University of Espírito Santo, Vitória, Brazil

8 Federal University of Rio Grande do Norte, Natal, Brazil

9 Federal University of Rio Grande do Sul, Porto Alegre, Brazil 